\title{
Empalamiento tóraco-abdominal: Reporte de un caso*
}

\author{
Drs. JUAN LUIS MORALES G. ${ }^{1}$, RENATO ARRIAGADA H. ${ }^{1}$, JUAN GÓMEZ P. ${ }^{2}$ \\ Departamento de Cirugía Hospital Clínico Herminda Martín. \\ Departamento de Anestesiología Hospital Clínico Herminda Martín. \\ 3 Interno Medicina Universidad Católica de la Santísima Concepción. \\ Chillán, Chile.
} Ints. ROBERTO SANTANA I. ${ }^{3}$, KATHERYN MARTEL E. ${ }^{3}$, CARLOS MORALES A. ${ }^{3}$

\section{Abstract}

\section{Impalement involving abdomen and thorax. Report of one case}

Impalements, especially those involving more than one anatomical region, are uncommon. We report a 30 years old male that was injured by a steel jimmy bar that entered by the left inguinal region and exited by the posterior part of the left hemithorax, causing multiple intestinal lesions, a lung and diaphragmatic lesion, costal and scapular fractures. He was operated, extracting the jimmy bar and correcting the multiple lesions. He was discharged ten days later.

Key words: Impalement, jimmy bar.

\section{Resumen}

Los casos de empalamiento son altamente infrecuentes, sobre todo aquellos que comprometen más de una región anatómica. Damos a conocer el caso de un obrero de 30 años que accidentalmente fue transfixiado por un chuzo, con entrada a nivel inguinal izquierdo y salida por hemitórax ipsilateral, lo que ocasionó múltiples lesiones intestinales, lesión diafragmática, lesión pulmonar y fracturas expuestas costales y escapular izquierda. Su evolución fue exitosa debido a un adecuado manejo pre e intrahospitalario.

Palabras clave: Empalamiento tóraco-abdominal.

\section{Introducción}

Se entiende como herida por empalamiento a toda herida penetrante causada por un objeto inciso-punzante, de forma que el objeto vulnerante queda incorporado en el cuerpo del sujeto ${ }^{1,2}$. El empalamiento es un evento espectacular cuyo tratamiento exitoso depende de un manejo precoz y bien estructurado, tanto en la etapa prehospitalaria como intrahospitalaria ${ }^{2,3}$.

El término "Empalamiento" fue originalmente popularizado en la época medieval, haciendo referencia al particular método por el cual las víctimas eran torturadas y asesinadas. El más famoso ejecutor de empalamientos fue el $15^{\circ}$ príncipe de Rumania Vlad Tepes, conocido como "Vlad el Empalador", o también entre sus contemporáneos como "Drácula" o "El hijo del Dragón"4.

*Recibido el 21 de Octubre de 2008 y aceptado para publicación el 19 de Enero de 2009.

Correspondencia: Dr. Juan L. Morales G.

Francisco Ramírez S/N, Chillán, Chile.

E-mail: jlmoralesg@ucsc.cl 
La espectacularidad y baja frecuencia de este trauma lleva a los autores a presentar este caso atendido en la unidad de urgencias del Hospital Clínico Herminda Martín de Chillán.

\section{Caso clínico}

Paciente de 30 años, sin antecedentes mórbidos conocidos. Trasladado desde Hospital de Yungay tras sufrir trauma tóraco-abdominal abierto por empalamiento luego de caer en pozo profundo de 12 metros y ser transfixiado por chuzo con entrada a nivel de región inguinal izquierda y salida por hemitórax ipsilateral por posterior, a nivel supraescapular (Figuras 1 y 2). Ingresa consciente, con aliento etílico, signos vitales estables y saturando $98 \%$ con oxígeno ambiental. Al examen físico destaca disminución del murmullo pulmonar en hemitórax izquierdo. Al ingreso a la unidad de emergencias, se soli- citan exámenes de laboratorio pertinentes, tomografía computarizada (TC) de Tórax, Abdomen y Pelvis, que mostró indemnidad de grandes vasos; lo que fue corroborado por ecotomografia, dada la interferencia del estudio tomográfico por el cuerpo extraño. Se indica cirugía de urgencia, realizando toracofrenolaparotomía paramediana izquierda amplia (Figura 3), encontrando lesión pulmonar en lóbulo inferior izquierdo transfixiante con hemoneumotórax de escasa cuantía, lesión diafragmática (Figura 4), cinco heridas transfixiantes de intestino delgado con lesión de sus mesos, y fractura expuesta de arco posterior de segunda costilla y escápula izquierda. Se extrae elemento traumático bajo visión directa realizando: neumorrafia, frenorrafia, reparación de lesiones intestinales con sus mesos, además de aseo quirúrgico de las fracturas. Finalmente, se realiza aseo prolijo de la cavidad abdominal y pleurotomía a caída libre. Se indica además aporte de volumen, analgesia y antibioticoterapia.

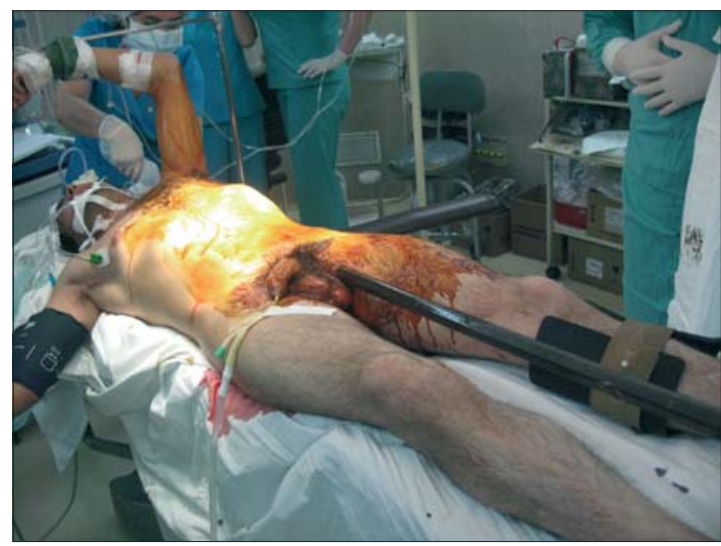

Figura 1. Sitio de entrada.

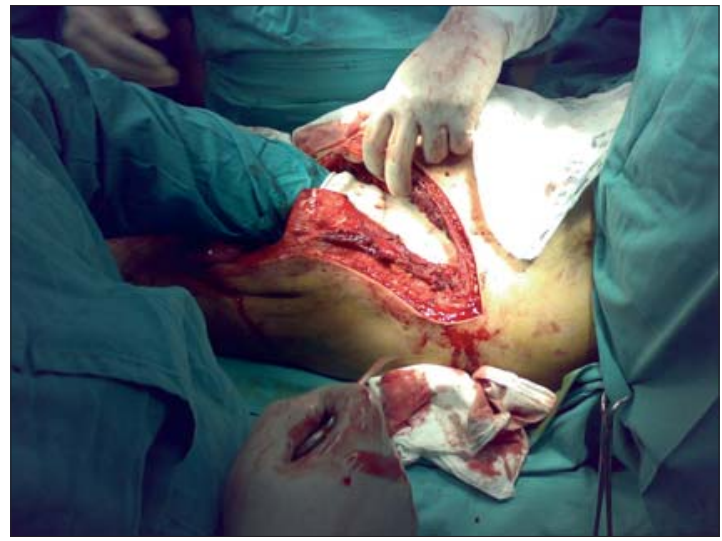

Figura 3. Toracofrenolaparotomía.

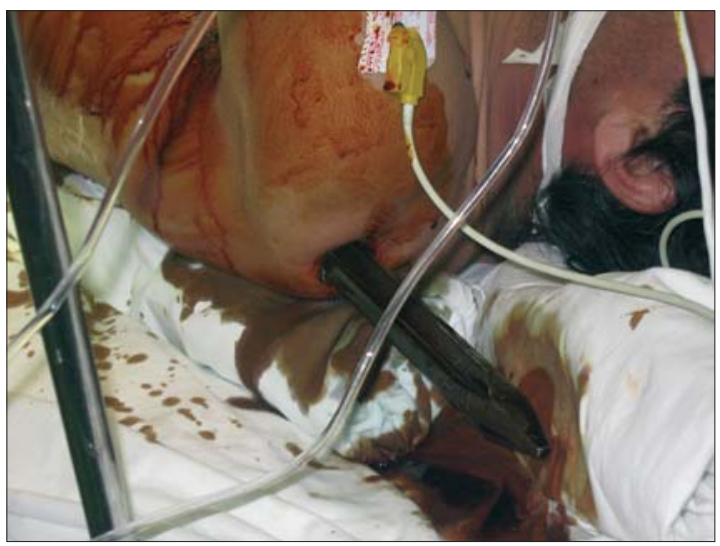

Figura 2. Sitio de salida.

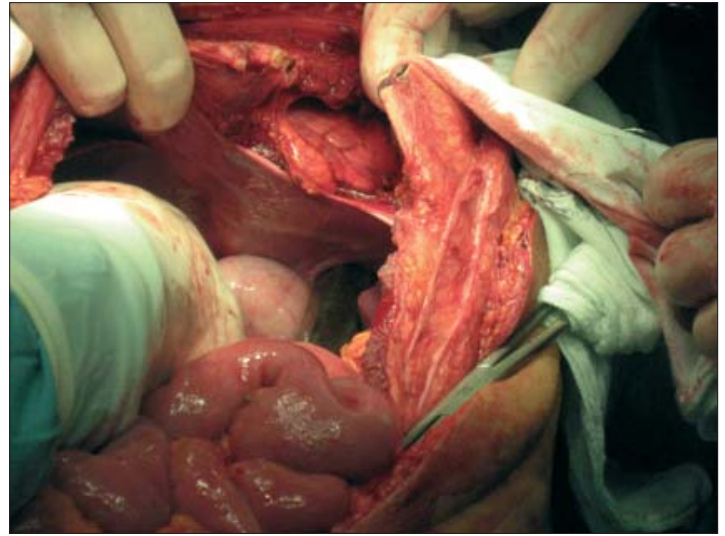

Figura 4. Aspecto de la incisión. 


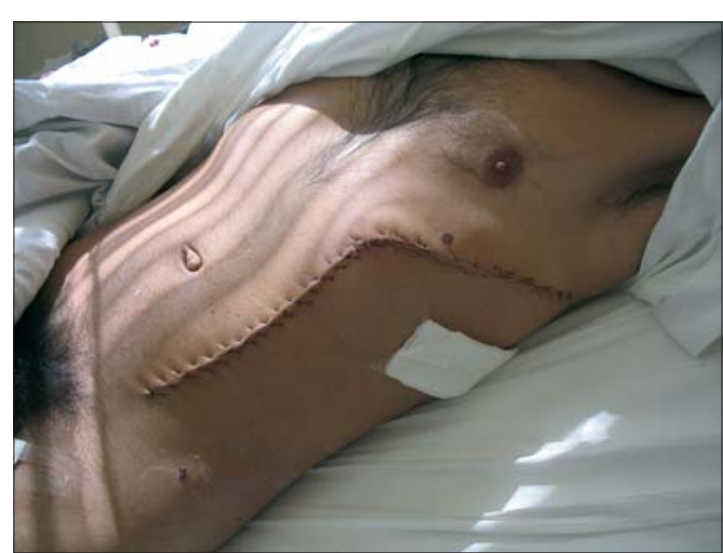

Figura 5. Incisión cerrada.

Realiza su postoperatorio en UCI evolucionando con estabilidad hemodinámica, conectado a ventilación mecánica las primeras 24 horas. Se traslada a sala de cirugía evolucionando satisfactoriamente, con herida operatoria en buenas condiciones (Figura 5), por lo que se otorga el alta luego de completar 10 días de tratamiento antibiótico, con indicación de control en su consultorio en una semana y control en policlínico de cirugía en un mes.

\section{Discusión}

El trauma por empalamiento tiene una baja incidencia. Son múltiples los mecanismos productores y la ingesta de alcohol y drogas juega un importante papel en su génesis. Existen reportes en la literatura de diversos sitios anatómicos de empalamiento: torácicos ${ }^{4-6}$, inguinales ${ }^{7}$, abdominales ${ }^{1-3}$, y otros.

Según Eachempati ${ }^{4}$, las lesiones por empalamiento pueden ser subdivididas en 2 grupos. El $1^{\text {er }}$ grupo (Tipo I) son las más frecuentes y resultan del impacto del cuerpo contra un objeto inmóvil, como en accidentes de tránsito o en accidentes laborales en faenas de construcción. El $2^{\text {do }}$ grupo (Tipo II) ocurren por manipulación intencional del cuerpo contra un objeto conocido, la mayoría de estas lesiones se encuentran en la región anogenital, en el contexto de perversiones sexuales.

La espectacularidad del trauma lleva muchas veces a actuar inadecuadamente, por lo que un adecuado manejo prehospitalario en conjunto con un precoz y estructurado manejo intrahospitalario son fundamentales para una correcta evolución. Las maniobras prehospitalarias se enfocan en dar un soporte vital adecuado y en el manejo de la herida. Se deben identificar y manejar lesiones que cursan con riesgo vital inmediato. Es importante no extraer el objeto, extraerlo suprimirá el efecto tapón ejercido sobre los vasos lesionados. Se debe estabilizar con compresas y controlar la hemorragia ejerciendo presión con las manos. El traslado debe ser rápido, eventualmente se necesitará acortar el objeto, para esto se deben usar herramientas de mano para un mayor control. La profilaxis antitetánica y la instauración de un esquema antibiótico son de regla. El manejo definitivo es el quirúrgico.

Se debe tener presente que esta clase de trauma involucra energía de gran intensidad, por lo que a las lesiones de un traumatismo abierto se deben sumar las de uno cerrado. Las características del objeto, la fuerza, la puerta de entrada, la dirección de la penetración y la ubicación de la punta darán la clave sobre qué órganos pueden estar lesionados antes de pasar a pabellón. El examen físico y el análisis del trayecto pueden ser complementados con ultrasonido, TC y angiografía si el estado del paciente lo permite. La exploración y la reparación de las lesiones se harán bajo principios quirúrgicos bien definidos. Es de vital importancia extraer el cuerpo extraño en la sala de operaciones bajo visión directa, para controlar y resolver en forma inmediata las diversas lesiones que se pueda encon$\operatorname{trar}^{6}$; la mayoría de las veces a través de laparotomías exploratorias, sin embargo, están descritos también procedimientos laparoscópicos diagnósticos en traumas abdominales por empalamientos ${ }^{8}$. Es necesaria una acuciosa revisión en busca de tejidos desvitalizados y un aseo prolijo donde se retirarán todos los cuerpos extraños, de esta forma se disminuirá al máximo la probabilidad de infección, principal complicación de esta clase de trauma.

Lo interesante de nuestro caso se basa en que el empalamiento compromete la región inguinal, abdominal y torácica y que, a través de una toracofrenolaparotomia paramediana izquierda amplia, pudimos ver y controlar todas las lesiones del paciente de manera adecuada.

En conclusión, el empalado es un paciente complejo, pero con un buen manejo extrahospitalario, un estructurado manejo intrahospitalario, profilaxis antitetánica y un esquema antibiótico adecuado puede evolucionar favorablemente.

\section{Referencias}

1. Guerra S. Actitud prehospitalaria ante el paciente empalado. Puesta al día emergencias y catástrofes 2000; 3: 168-173.

2. Lasagna R, Lasagna N, Fariña C, Fariña L. Empalamiento transabdominal. Rev Chil Cir 2002; 4: 408410.

3. Yavrouian R, Mahmoud A, Matolo N. Abdominal 
impalement resulting in an isolated duodenal injury. Surg Rounds 2008; 2: 59-61.

4. Eachempati S. Impalement injuries. Duke Trauma Newsletter 1998; 2: 6-7.

5. Hernández J, Mejides R, Pérez de León R, Hernández M. Inusual cuerpo extraño intratorácico. Rev Cubana Cir 2007; 46: 10-16.

6. Darbari A, Tandon S, Kumar A. Thoracic impalement injuries. IJTCVS 2005; 21: 229-231.

7. Baeza C, Medellín U, Domínguez T, Atzín J, García L. Empalamiento inguinal. Presentación de dos casos clínicos. Cir Ciruj 2008; 76: 83-86.

8. Wiesel O, Makrin V, Lubezky N, Klausner J, Schulman C, Soffer D. Diagnostic laparoscopy for the evaluation of abdominal impalement injuries. IMAJ 2008; 10: 314-315. 